\title{
Interlayer exchange coupling: Preasymptotic corrections
}

\author{
P. Brund: \\ Max-Planck-Institut für Mikrostrukturphysik, Weinberg 2, D-06120 Halle, Germany
}

(7 January 1999)

\begin{abstract}
In the asymptotic limit, the interlayer exchange coupling decays as $D^{-2}$, where $D$ is the spacer thickness. A systematic procedure for calculating the preasymptotic corrections, i.e., the terms of order $D^{-n}$ with $n \geq 3$, is presented. The temperature dependence of the preasymptotic corrections is calculated. The results are used to discuss the preasymptotic corrections for the $\mathrm{Co} / \mathrm{Cu} / \mathrm{Co}(001)$ system.
\end{abstract}

Published in: Eur. Phys. J. B 11, 83-89 (1999)

\section{INTRODUCTION}

Since its discovery in 1990 the phenomenon of oscillatory interlayer exchange coupling has been the subject of an intense experimental and theoretical activity.2. Its mechanism is now well understood and can be attributed to the quantum size effect due to spin-dependent confinement of electrons in the non-magnetic spacer layer. 10

A remarkable result of the theory of interlayer exchange coupling is that it becomes particularly simple in the limit of large spacer thickness $D$ (asymptotic regime): (i) the periods of oscillations versus spacer layer thickness are uniquely determined by stationary spanning vectors of the bulk Fermi surface of the spacer material, 8 (ij) the oscillation amplitude has a universal $D^{-2}$ decayl (except for special nested Fermi surfaces which give rise to a $D^{-3 / 2}$ or $D^{-1}$ decay law 10 ), (iii) the amplitudes and phases of the oscillatory components are determined respectively by the modules and arguments of the spin asymmetry of the (complex) electron reflection coefficients $g$ n the ferromagnetic layers bounding the spacer layer.

Although these simple rules stricly speaking hold only in the limit of infinite spacer thickness, they proved to be extremely successful in explaining and predicting the interlayer exchange coupling observed experimentally for spacer thicknesses down to less than 10 atomic layers (AL).11.12 The surprisingly good results of the asymptotic approximation are also confirmed by first-principles calculations. 13.16

The exact expression of the interlayer exchange coupling may be expanded in a series of powers of $1 / D$. The first non-zero term is the $D^{-2}$ term; it corresponds to the asymptotic approximation. The higher order terms (varying like $D^{-n}$ with $n \geq 3$ ) are called the preasymptotic corrections.

The overall success of the asymptotic approximation in explaining the experimental results obtained at fairly low spacer thicknesses is a priori surprising and raises the question of a quantitative determination of the range of validity of the asymptotic approximation. In addition, there are also some cases where the asymptotic approximation fails in providing an accurate description of the interlayer coupling for small spacer thicknesses.
The inadequacy of the asymptotic approximation for an accurate description of the interlayer exchange coupling is best illustrated by the $\mathrm{Co} / \mathrm{Cu} / \mathrm{Co}(001)$ system. The "exact" calculations (i.e., not relying on the asymptotic approximation) show that the $D^{-2}$ decay law of short period oscillation is not obeyed until the spacer thickness reaches approximately 20 AL.17 In addition, the amplitude of the long period oscillation for $\mathrm{Co} / \mathrm{Cu} / \mathrm{Co}(001)$ with thick Colazers as calculated from the asymptotic approximation 1823 is typically at least one order of magnitude too small as compared to the one obtained from calculation do not rely on the asymptotic approximation.16.17 The inadequacy of the asymptotic approximation to describe accurately the long period oscillation of the $\mathrm{Co} / \mathrm{Cu} / \mathrm{Co}(001)$ system with thick Co layers is also illustrated by the fact that the corresponding amplitude, as well as the period itself depends on the $\mathrm{Cu}$ thickness range which is used to determine it 2724 and usually does not agree with the one calculated from the bulk $\mathrm{Cu}$ Fermi surface.25

The present paper is devoted to a detailled discussion of the validity of the asymptotic approximation and of the preasymptotic corrections. It is organized as follows. First, in Sec. II I recall the assumptions underlying the asymptotic approximation, and the results that it yields. Next, in Sec. III I present a systematic method for calculating the preasymptotic corrections to arbitrary order and their temperature dependence, and we carry out explicitely the calculation of the first preasymptotic correction, i.e., the $D^{-3}$ term. The Sec. IV is devoted to the discussion of the results with emphasis on the $\mathrm{Co} / \mathrm{Cu} / \mathrm{Co}(001)$ system, and concluding remarks are given in Sec. $\mathrm{V}$.

\section{ASYMPTOTIC APPROXIMATION}

is $\mathrm{U}^{\mathrm{T}}$

$$
\begin{aligned}
J(D, T)= & -\operatorname{Im} \int_{-\infty}^{+\infty} \mathrm{d} \varepsilon f(\varepsilon, T) \\
& \times \int \mathrm{d}^{2} \mathbf{k}_{\|} M\left(\varepsilon, \mathbf{k}_{\|}\right) \mathrm{e}^{\mathrm{i} q\left(\varepsilon, \mathbf{k}_{\|}\right) D}
\end{aligned}
$$


In this expression, $q\left(\varepsilon, \mathbf{k}_{\|}\right) \equiv k_{z}^{+}-k_{z}^{-}$is the difference between wave-vectors of an electron propagating through the spacer layer in the $+z$ and $-z$ directions (the $z$ axis is taken perpendicular to the layer plane). Here a single contribution has been considered; in the general case there would be several such contributions, due to multiple bands in the spacer materials and to higher harmonics (i.e., higher order terms in an expansion in powers of $\left.\mathrm{e}^{\mathrm{i} q\left(\varepsilon, \mathbf{k}_{\|}\right)}\right)$but the calculation of the various contributions is exactly the same; thus, for the sake of simplicity, a single contribution is considered here. As explicitely indicated, $q\left(\varepsilon, \mathbf{k}_{\|}\right)$varies with the energy $\varepsilon$ and with the in-plane wave-vector $\|$. The other factor in the integrand of Eq. (11) are the Fermi-Dirac function $f(\varepsilon, T)$ and the complex amplitude $M\left(\varepsilon, \mathbf{k}_{\|}\right)$which depends on the spinasymmetry of the reflection coefficients at the spacerferromagnet interfaces. 6 The range of the integration over $\mathbf{k}_{\|}$in Eq. (1]) is the surface Brillouin zone corresponding to the crystalline orientation of the layers.

The asymptotic approximation is based upon the observation that, because of the strong variation of the Fermi-Dirac function at the Fermi energy, and because of the rapid variation with $\varepsilon$ and $\mathbf{k}_{\|}$of the exponential factor, the behavior of Eq. (11) at large $D$ is dominated by the contribution of states on the Fermi surface, such that the spanning vector of the Fermi surface, $q\left(\varepsilon, \mathbf{k}_{\|}\right)$, is stationary with respect to $\mathbf{k}_{\|}$In the general case, there may several such stationary spanning vectors, each of them giving rise to an oscillatory component of the interlayer exchange coupling, but for simplicity, I shall consider the case of a single component; the generalization to the case of multiple components is immediate.

The in-plane wave-vector $\mathbf{k}_{\|}^{\star}$ corresponding to the stationary spanning vector of the Fermi surface $q^{\star}$ is taken as the origin for $\mathbf{k}_{\|}$and the Fermi level is taken as the origin for $\varepsilon$.

The variation of $M\left(\varepsilon, \mathbf{k}_{\|}\right)$with $\varepsilon$ and $\mathbf{k}_{\|}$is neglected, i.e., we assume

$$
M\left(\varepsilon, \mathbf{k}_{\|}\right) \approx M_{0 ; 0,0}
$$

where $M_{0 ; 0,0}$ is a constant (the motivation for the choice of the notations will appear clearly below). Further, we expand $q\left(\varepsilon, \mathbf{k}_{\|}\right)$near $q^{\star}$ as

$$
q\left(\varepsilon, \mathbf{k}_{\|}\right) \approx q^{\star}+\frac{2 \varepsilon}{\hbar v_{F}}-\frac{k_{x}{ }^{2}}{\kappa_{x}}-\frac{k_{y}{ }^{2}}{\kappa_{y}},
$$

where $v_{F}$ is the Fermi velocity, and $\kappa_{x}$ and $\kappa_{y}$ the curvature radii of the Fermi surface corresponding to $\mathbf{k}_{\|}^{\star}$ (the $x$ and $y$ axes are chosen so as to eliminate the term proportional to $k_{x} k_{y}$ ). Finally, since only the neighbohood of $\mathbf{k}_{\|}^{\star}$ contributes significantly to the integral, we extend the integration range for $k_{x}$ and $k_{y}$ from $-\infty$ to $+\infty$. Then, we obtain easily

$$
J(D, T) \approx \operatorname{Im}\left[\frac{\mathrm{e}^{\mathrm{i} q^{\star} D}}{D^{2}} A_{0}(D T)\right]
$$

with the complex amplitude determined by

$$
\begin{aligned}
A_{0}(D T) \equiv & \frac{\pi}{2} \hbar v_{F}\left(\kappa_{x}\right)^{1 / 2}\left(\kappa_{y}\right)^{1 / 2} M_{0 ; 0,0} \\
& \times F_{0}\left(\frac{2 \pi k_{B} T D}{\hbar v_{F}}\right) .
\end{aligned}
$$

The temperature dependence is given by the function

$$
F_{0}(x) \equiv \frac{x}{\sinh x} .
$$

The predictions of the asymptotic approximation are summarized as follows: (i) at $T=0$ the coupling is given by a periodic function of $D$ multiplied by a decay factor $D^{-2}$, (ii) the phase of the oscillations is independent of the temperature, (iii) the complex amplitude factor varies with spacer thickness and temperature only as a function of the product $D T$.

\section{CALCULATION OF THE PREASYMPTOTIC CORRECTIONS}

Let us compute the preasymptotic corrections that appear when we release the approximations made in the previous section. First, instead of assuming that $M\left(\varepsilon, \mathbf{k}_{\|}\right)$is a constant, we expand it around $\varepsilon_{F} \equiv 0$ and $\mathbf{k}_{\|}^{\star} \equiv 0$ as

$$
M\left(\varepsilon, \mathbf{k}_{\|}\right) \equiv \sum_{p, q, r \geq 0} M_{p ; q, r} \varepsilon^{p} k_{x}^{q} k_{y}^{r},
$$

which defines the expansion coefficients $M_{p ; q, r}$. In addition, we extend the expansion of $q\left(\varepsilon, \mathbf{k}_{\|}\right)$beyond the first order in $\varepsilon$ and the second order in $k_{x}$ and $k_{y}$ :

$$
\begin{gathered}
q\left(\varepsilon, \mathbf{k}_{\|}\right)=q^{\star}+\frac{2 \varepsilon}{\hbar v_{F}}-\frac{k_{x}{ }^{2}}{\kappa_{x}}-\frac{k_{y}{ }^{2}}{\kappa_{y}}+Q\left(\varepsilon, \mathbf{k}_{\|}\right) \\
Q\left(\varepsilon, \mathbf{k}_{\|}\right) \equiv \sum_{2 s+t+u>2} Q_{s ; t, u} \varepsilon^{s} k_{x}{ }^{t} k_{y}{ }^{u}
\end{gathered}
$$

which defines the expansion coefficients $Q_{s ; t, u}$. The factor $\mathrm{e}^{\mathrm{i} q D}$ (the $\varepsilon$ and $\mathbf{k}_{\|}$arguments will be dropped in the following) in Eq. (11) is then rewritten as

$$
\begin{aligned}
\mathrm{e}^{\mathrm{i} q D}= & \mathrm{e}^{\mathrm{iq}{ }^{\star} \mathrm{D}} \exp \left(\frac{\mathrm{i} \varepsilon D}{\hbar v_{F}}\right) \exp \left(\frac{-\mathrm{i} k_{x} D}{\kappa_{x}}\right) \exp \left(\frac{-\mathrm{i} k_{y} D}{\kappa_{y}}\right) \\
& \times \sum_{n \geq 0} \frac{(i Q D)^{n}}{n !} .
\end{aligned}
$$

Then the product $M\left(\varepsilon, \mathbf{k}_{\|}\right.$with the last factor of the above equation is expanded in powers of $\varepsilon, k_{x}$ and $k_{y}$ as follows

$$
M \sum_{n \geq 0} \frac{(i Q D)^{n}}{n !}=\sum_{p, q, r \geq 0} C_{p ; q, r}(D) \varepsilon^{p} k_{x}{ }^{q} k_{y}{ }^{r},
$$


which defines the new expansion coefficients $C_{p: q, r}(D)$. Their explicit expression in terms of the coefficients $M_{p ; q, r}$ and $Q_{s ; t, u}$ can be obtained by a straighforward term-by-term identification.

Inserting the above expressions in Eq. (11), we obtain

$$
\begin{aligned}
J(D, T)= & -\operatorname{Im}\left[\mathrm{e}^{\mathrm{i} q^{\star} D} \sum_{p, q, r \geq 0} C_{p ; q, r}(D)\right. \\
& \times \int_{-\infty}^{+\infty} \mathrm{d} \varepsilon \varepsilon^{p} f(\varepsilon, T) \exp \left(\frac{\mathrm{i} \varepsilon D}{\hbar v_{F}}\right) \\
& \times \int_{-\infty}^{+\infty} \mathrm{d} k_{x} k_{x}{ }^{q} \exp \left(\frac{-\mathrm{i} k_{x}{ }^{2} D}{\kappa_{x}}\right) \\
& \left.\times \int_{-\infty}^{+\infty} \mathrm{d} k_{y} k_{y}{ }^{r} \exp \left(\frac{-\mathrm{i} k_{y}{ }^{2} D}{\kappa_{y}}\right)\right]
\end{aligned}
$$

The above expression is non-zero only if $q$ and $r$ are even. Performing the integrations as explained in the Appendix, we obtain

$$
\begin{aligned}
J(D, T)= & \operatorname{Im}\left[\frac{\mathrm{e}^{\mathrm{i} q^{\star} D}}{D^{2}} \frac{\pi}{2} \hbar v_{F}\left(\kappa_{x}\right)^{1 / 2}\left(\kappa_{y}\right)^{1 / 2}\right. \\
& \times \sum_{p, q, r \geq 0} C_{p ; 2 q, 2 r}(D) \frac{1}{D^{p+q+r}} \\
& \times \frac{(-1)^{q+r} \mathrm{i}^{p+q+r}}{2^{p+q+r}} p ! \frac{(2 q+1) ! !}{2 q+1} \frac{(2 r+1) ! !}{2 r+1} \\
& \left.\times\left(\hbar v_{F}\right)^{p} \kappa_{x}^{q} \kappa_{y}^{r} F_{p}\left(\frac{2 \pi k_{B} T D}{\hbar v_{F}}\right)\right],
\end{aligned}
$$

with

$$
(2 n+1) ! ! \equiv \frac{(2 n+1) !}{2^{n} n !},
$$

and where the functions $F_{n}(x)$ governing the temperature dependence of the coupling are defined by

$$
F_{n}(x) \equiv \frac{(-1)^{n}}{n !} x^{n+1} \frac{\mathrm{d}^{n}}{\mathrm{~d} x^{n}}\left(\frac{1}{\sinh x}\right) .
$$

Finally, we reorder the terms of Eq. (13) so as to express it as a series in powers of $1 / D$ :

$$
J(D, T)=\operatorname{Im}\left[\frac{\mathrm{e}^{\mathrm{i} q^{\star} D}}{D^{2}} \sum_{n \geq 0} \frac{A_{n}(D T)}{D^{n}}\right] .
$$

The expressions of the functions $A_{n}(D T)$ in terms of the coefficients $M_{p ; q, r}$ and $Q_{s ; t, u}$ are obtained from Eq. (13) and from the explicit expression of the expansion coefficients $C_{p ; q, r}(D)$ after tedious but straightforward algebraic manipulations. Below, I give the explicit expression of the asymptotic approximation term $A_{0}$ and of the first preasymptotic correction term $A_{1}$ :

$$
A_{0}=\frac{\pi}{2} \hbar v_{F}\left(\kappa_{x}\right)^{1 / 2}\left(\kappa_{y}\right)^{1 / 2} M_{0 ; 0,0} F_{0}
$$

$$
\begin{aligned}
A_{1}= & \frac{\pi}{2} \hbar v_{F}\left(\kappa_{x}\right)^{1 / 2}\left(\kappa_{y}\right)^{1 / 2} \\
\times & \left\{-\frac{\mathrm{i}}{2}\left(M_{0 ; 2,0} \kappa_{x}+M_{0 ; 0,2} \kappa_{y}\right) F_{0}\right. \\
- & \frac{3}{4}\left[\left(M_{0 ; 0,0} Q_{0 ; 4,0}+M_{0 ; 1,0} Q_{0 ; 3,0}\right) \kappa_{x}^{2}\right. \\
& \left.+\left(M_{0 ; 0,0} Q_{0 ; 0,4}+M_{0 ; 0,1} Q_{0 ; 0,3}\right) \kappa_{y}^{2}\right] F_{0} \\
- & \frac{1}{4}\left(M_{0 ; 0,0} Q_{0 ; 2,2}+M_{0 ; 1,0} Q_{0 ; 1,2}\right. \\
& \left.+M_{0 ; 0,1} Q_{0 ; 2,1}\right) \kappa_{x} \kappa_{y} F_{0}+\frac{i}{2} M_{1 ; 0,0} \hbar v_{F} F_{1} \\
+ & \frac{1}{4}\left[\left(M_{0 ; 0,0} Q_{1 ; 2,0}+M_{0 ; 1,0} Q_{1 ; 1,0}\right) \hbar v_{F} \kappa_{x}\right. \\
& \left.+\left(M_{0 ; 0,0} Q_{1: 0,2}+M_{0 ; 0,1} Q_{1 ; 0,1}\right) \hbar v_{F} \kappa_{y}\right] F_{1} \\
- & \left.\frac{1}{2} M_{0 ; 0,0} Q_{2 ; 0,0}\left(\hbar v_{F}\right)^{2} F_{2}\right\} .
\end{aligned}
$$

\section{DISCUSSION}

\section{A. Symmetry considerations}

As discussed in detail in Ref. 7, the various critical points $\mathbf{k}_{\|}^{\star}$ of the two-dimensional Brillouin zone giving the oscillatory contributions to the interlayer exchange coupling can classified according to their symmetry: following the terminology introduced in Ref. 7, critical points corresponding to high-symmetry points of the twodimensional Brillouin zone are termed essential, critical points located on high-symmetry lines are termed semiessential, while critical points possessing no particular symmetry are termed accidental.

The symmetry considerations impose a number of restrictions on the c oefficients $M_{p ; q, r}, Q_{s ; t, u}$ and $C_{p ; q, r}$. These are given below:

(i) For an essential critical point, we have $M_{p ; q, r}=0$, $Q_{p ; q, r}=0$ and $C_{p ; q, r}=0$ if $q$ or $r$ is odd. If in addition, the critical point corresponds to a rotation axis of order equal to or larger than 3 , then $\kappa_{x}=\kappa_{y}, M_{p ; 2 q, 2 r}=$ $M_{p ; 2 r, 2 q}, Q_{p ; 2 q, 2 r}=Q_{p ; 2 r, 2 q}$, and $C_{p ; 2 q, 2 r}=C_{p ; 2 r, 2 q}$.

(ii) For a semi-essential critical point (without restriction, we chose the $y$ axis parallel to the high-symmetry line), we have $M_{p ; q, r}=0, Q_{p ; q, r}=0$ and $C_{p ; q, r}=0$ if $q=0$.

(iii) For an accidental critical point, no restriction applies.

\section{B. Preasymptotic corrections at $T=0$}

If we retain only the first preasymptotic correction term in Eq. (16), the coupling may be expressed as

$$
J \approx \operatorname{Im}\left[\frac{\mathrm{e}^{\mathrm{i} q^{\star} D}}{D^{2}} A_{0}\left(1+\frac{\Delta}{D}\right)\right],
$$


where the complex length

$$
\Delta \equiv \frac{A_{1}}{A_{0}}
$$

characterizes the preasymptotic correction. Thus, for $D \gg|\Delta|$, one has

$$
J \approx \frac{\left|A_{0}\right|}{D^{2}}\left(1+\frac{|\Delta| \cos \delta}{D}\right) \sin \left(q^{\star} D+\phi+\frac{|\Delta| \sin \delta}{D}\right),
$$

where $\phi$ and $\delta$ are respectively the arguments of $A_{0}$ and $\Delta$. From the above equation, it appears that the real part of $\Delta$ yields a correction of the amplitude of the oscillatory coupling; depending on whether $\cos \delta$ is positive or negative, one has an increase or a decrease of the amplitude. On the other hand, the imaginary part of $\Delta$ contributes to a correction of the phase of the oscillation; i.e., for low spacer thickness, the apparent period of the oscillations differs from the asymptotic one 26

$$
\Lambda^{\star} \equiv \frac{2 \pi}{\left|q^{\star}\right|}
$$

and becomes

$$
\Lambda_{\mathrm{app}} \approx \Lambda^{\star}\left(1-\frac{|\Delta| \sin \delta}{q^{\star} D^{2}}\right) ;
$$

depending on whether $\sin \delta$ and $q^{\star}$ are of the same sign or not, one as a decrease or an increase of the oscillation period.

Finally, the above discussion shows that a quantitative criterion for the validity of the asymptotic approximation is given by two following conditions

$$
\begin{aligned}
& D \gg|\Delta| \cos \delta, \\
& D \gg\left(\frac{|\Delta||\sin \delta|}{\left|q^{\star}\right|}\right)^{1 / 2} .
\end{aligned}
$$

\section{Temperature dependence of the preasymptotic corrections}

The temperature dependence of the coupling is governed by the functions $F_{n}(x)$ defined by Eq. (15). The functions $F_{n}(x)$ with $n=0-3$ are given explicitely below:

$$
\begin{aligned}
& F_{0}(x)=\frac{x}{\sinh x} \\
& F_{1}(x)=\frac{x^{2}}{\sinh ^{2} x} \cosh x \\
& F_{2}(x)=\frac{x^{3}}{\sinh ^{3} x}\left(1+\frac{\sinh ^{2} x}{2}\right) \\
& F_{3}(x)=\frac{x^{4}}{\sinh ^{4} x} \cosh x\left(1+\frac{\sinh ^{2} x}{6}\right) .
\end{aligned}
$$

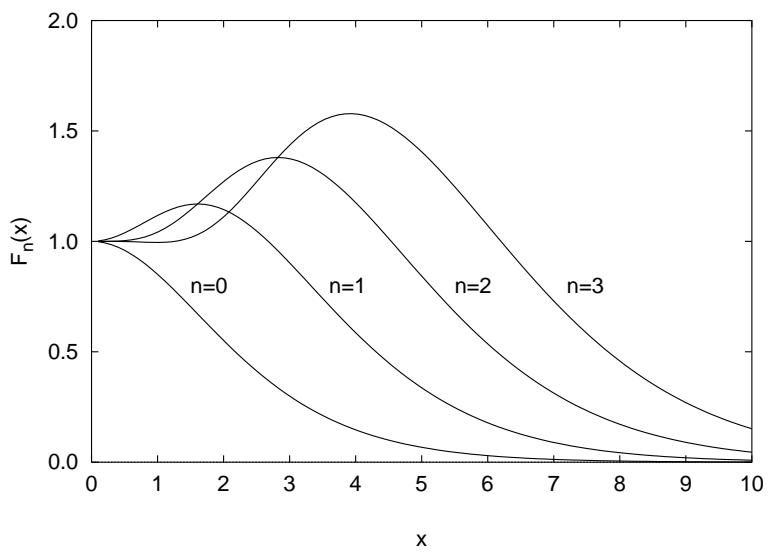

FIG. 1. Plot of the functions $F_{n}(x)$ with $n=0-3$.

They are displayed on Fig. 1. The functions $F_{n}(x)$ have the following general properties:

$$
\begin{aligned}
& F_{n}(x) \approx 1+\beta_{n} x^{2} \text { for } x \ll 1, \\
& F_{n}(x) \approx \frac{2}{n !} x^{n+1} \mathrm{e}^{-x} \text { for } x \gg n+1,
\end{aligned}
$$

where the constants $\beta_{n}$ are given by

$$
\begin{aligned}
& \beta_{0}=-\frac{1}{6}, \\
& \beta_{1}=\frac{1}{6}, \\
& \beta_{n}=0 \text { for } n \geq 2 ;
\end{aligned}
$$

$F_{n}(x)$ with $n \geq 1$ has a maximum at

$$
\begin{aligned}
x_{n}^{\star} & \approx n+1, \text { and } \\
F_{n}\left(x_{n}^{\star}\right) & \approx \frac{2}{n !}\left(\frac{n+1}{\mathrm{e}}\right)^{n+1}
\end{aligned}
$$

Because of the marked difference between $F_{0}(x)$ and the functions $F_{n}(x)$ with $n \geq 1$, the temperature dependence of the preasymptotic corrections is in general not the same as the one of the asymptotic term. In other words, the parameter $\Delta$ characterized the preasymptotic corrections defined in Sec. IVB generally varies with the temperature; thus, the extension of the asymptotic regime may depend on the temperature. As expected and appears clearly from Eq. (17b), the temperature dependence of $\Delta$ arises from the terms proportional to $F_{1}$ and $F_{2}$, i.e. from the corrections due to the $\varepsilon$ variation of $M\left(\varepsilon, \mathbf{k}_{\|}\right)$and $Q\left(\varepsilon, \mathbf{k}_{\|}\right)$.

\section{Comparison with previous work}

The question of preasymptotic corrections has been addressed previously by Mathon et al. 19.22 .23 They have considered the correction due to the energy dependence 
of the argument of $M\left(\varepsilon, \mathbf{k}_{\|}\right)$. This correspond to assume that the only non-zero correction parameter is $M_{1 ; 0,0}$ and that the ratio $M_{1 ; 0,0} / M_{0 ; 0,0}$ is purely imaginary. In this case, they find that the preasymptotic corrections modify the amplitude and the temperature dependence of the coupling. This conclusion is in agreement with the result of the present paper, if we restrict our analysis to the same assumptions. However, even within the restrictive assumptions, the method used by Mathon et al. is correct only to the order $D^{-3}$ because the the terms order $D^{-n}$ with $(n \geq 4)$ which are neglected are of the same magnitude as the ones which are included.

Furthermore, as the present analysis shows, there are other sources of preasymptotic corrections, for example the $\mathbf{k}_{\|}$variation of $M\left(\varepsilon, \mathbf{k}_{\|}\right)$, that a priori, are equally important as the ones considered by Mathon et al.

\section{E. Discussion of the $\mathrm{Co} / \mathrm{Cu} / \mathrm{Co}(001)$ case}

We shall now focus on the particular case of the $\mathrm{Co} / \mathrm{Cu} / \mathrm{Co}(001)$ system, which is often considered as a model system for the problem of interlayer exchange coupling.

\section{Summary of the available data}

Let us first recall the predictions of the asymptotic approximation for this system. The most striking result of the asymptotic approximation here is that the interlayer exchange coupling comprises a long-period oscillation originating for the center $\bar{\Gamma}$ of the two-dimensional Brillouin zone (essential critical point) and of a short-period oscillation originating from 4 equivalent semi-essential critical points located on the $\bar{\Gamma}-\bar{X}$ lines $;$ this prediction is confirmed unambiguously both by experiments 27 and by "exact" (i.e., not relying on the asymptotic approximation) calculations, 1317 and the periods are in good agreement with the ones predicted by the asymptotic approximation. In the following, quantities related to the short-period and long-period components will labelled by $S$ and $L$ indices, respectively.

Quite generally, the function $M\left(\varepsilon_{\text {d }} \mathrm{k}_{\|}\right)$determining the strength of the coupling is given by

$$
M\left(\varepsilon, \mathbf{k}_{\|}\right) \equiv \frac{\left(r^{\uparrow}-r^{\downarrow}\right)^{2}}{8 \pi^{3}},
$$

where $r^{\uparrow}\left(r^{\downarrow}\right)$ is the reflection coefficient for electrons with the spin parallel (antiparallel) to the majority spin of Co. Since the majority spin band structure of Co is very close to the $\mathrm{Cu}$ band structure, one has $r^{\uparrow} \approx 0$ and it is sufficient to consider $r^{\downarrow}$.

For the short period oscillation, one has total reflection for minority spin electron, due to a local gap (for the bands of relevant symmetry) in the minority spin band structure of Co, i.e., $\left|r_{S}^{\downarrow}\right|=1.1923$ As a consequence, the amplitude of the short period oscillation is large and depends only very weakly upon the $\mathrm{C}$ thickness. These facts are confirmed by "exact" calculations.13 17 The period of oscillation obtained from an "exact" calculation, $\left(\Lambda_{S}=2.53 \mathrm{AL}\right)^{24}$ is in excellent agreement with the one calculated within the asymptotic approximation with the same set of potential parameters $\left(\Lambda_{S}=2.50 \mathrm{AL}\right) 25$ furthermore, the apparent period agrees very well with the asymptotic one down to $\mathrm{Cu}$ thicknesses as low a $5 \mathrm{AL}$. On the other hand, as appears very clearly from the Fig. 3a of Ref. 17, the asymptotic $D^{-2}$ decay law is satisfied only for $\mathrm{Cu}$ spacer thicknesses larger $20 \mathrm{AL}$; in practice, this means that for a quantitative comparison with experiment results (which are usually obtained for spacer ticknesses smaller than $20 \mathrm{AL}$ ), the amplitude calculated from the asymptotic approximation is inappropriate.

For the long period oscillation, the situation is quite different. Here, the reflection coefficient $r \downarrow$ is quite small for a semi-infinite Co layer $\left(\left|r_{L}^{\downarrow}\right| \approx 0.15\right)$ 目 18 . 20, 21, 28 which leads to a very small amplitude for the long-period oscillatory component; all theoretical calculation, whether "asymptotic" 1823 or "exact" $13-17$ agree on this point. In addition, there is a strong variation of the amplitude of the long period oscillation with Co layer thickness which is due to quantum interferences in the Co layer, 29 a prediction which has been confirmed experimentally 30 and by "exact" theoreticalcalgulations.14 172.28 However, although both asymptotic 1823 and "exact" 14 17 calculations agree on the fact that the long period oscillation has a very weak amplitude for thick Co layers, they disagree on the value of the amplitude: while "exact" calculation yield an amplitude of the long period oscillation of the order of $10 \%$ of the short period amplitude 13,16.17 asymptotic calculations yield a ratio of the order of $1 \%$ only 1923 In addition, the value of the long period coupling obtained from "exact" calculations, $\Lambda_{L}=5.09 \mathrm{AL}$,24 differs markedly from the one calculated from the asymptotic approximation with the same set of potential parameters, $\Lambda_{L}=6.49 \mathrm{AL} 25$ and depends on the thickness range which is used to determine it as is seen from Fig. 2 of Ref. 17.

So, our purpose is to explain why the asymptotic approximation works well for some aspects of the coupling and departs markedly from "exact" calculations for some other aspects.

For both the long-period and short-period contribution, the preasymptotic corrections due to the $\varepsilon$ and $\mathbf{k}_{\|}$ dependence of $Q\left(\varepsilon, \mathbf{k}_{\|}\right)$is unimportant, so that we shall focus on the corrections due the variation of $M\left(\varepsilon, \mathbf{k}_{\|}\right)$. Thus, by taking the symmetry considerations mentioned in Sec. IVA, the corrections are given by

$$
\begin{aligned}
& \Delta_{L}=-\mathrm{i} \frac{M_{0 ; 2,0}^{L}}{M_{0 ; 0,0}^{L}} \kappa_{x}^{L}+\frac{\mathrm{i}}{2} \frac{M_{1 ; 0,0}^{L}}{M_{0 ; 0,0}^{L}} \hbar v_{F}^{L} \frac{F_{1}^{L}}{F_{0}^{L}}, \\
& \Delta_{S}=-\frac{\mathrm{i}}{2}\left(\frac{M_{0 ; 2,0}^{S}}{M_{0 ; 0,0}^{S}} \kappa_{x}^{S}+\frac{M_{0 ; 0,2}^{S}}{M_{0 ; 0,0}^{S}} \kappa_{y}^{S}\right)
\end{aligned}
$$




$$
+\frac{\mathrm{i}}{2} \frac{M_{1 ; 0,0}^{S}}{M_{0 ; 0,0}^{S}} \hbar v_{F}^{S} \frac{F_{1}^{S}}{F_{0}^{S}}
$$

respectively. Let us now recall that within elementary models such as the free-electron model or the single-band tight-binding model, the reflection coefficient for a semiinfine barrier is either a real number of module smaller than 1 (partial reflection) or a complex number of module equal to 1 (total reflection). As shown in Ref. G, this remains approximately true for realistic multi-band systems under rather general circumstances.

\section{Short-period oscillation}

Thus, for the short period oscillation, only the phase of the reflection coefficient varies with $\varepsilon$ and $\mathbf{k}_{\|}$, and as a consequence, $\Delta_{S}$ is a real number. Hence, according to the discussion of Secs. IVB and IV , the preasymptotic correction affects only the amplitude of the coupling and its temperature dependence, while the period is not affected by the preasymptotic corrections; these conclusions are in agreement with the results summarized above. Similar conclusions have been obtained by Mathon et al.23 who have considered the preasymptotic correction associated with the energy dependence $M\left(\varepsilon, \mathbf{k}_{\|}\right)$, i.e., with $M_{0 ; 0,1}^{S}$ and neglected the one associated with its $\mathbf{k}_{\|}$dependence, i.e., with $M_{0: 2,0}^{S}$ and $M_{0 ; 0,2}^{S}$; indeed in view of the similarity of their corrected results (see Fig. 13 of Ref. 23) with those of "exact" calculations (see Fig. 3 of Ref. 17), it seems that the $M_{0 ; 0,1}^{S}$ term already accounts for a large part of the total preasymptotic correction. Further work would be needed to assess the importance of the $M_{0 ; 2,0}^{S}$ and $M_{0 ; 0,2}^{S}$ terms.

\section{Long-period oscillation}

For the long-period oscillation, on the other hand, since $r_{L}^{\downarrow}$ is real, only the magnitude varies with $\varepsilon$ and $\mathbf{k}_{\|}$and the phase is constant; thus, $\Delta_{L}$ is purely imaginary. As discussed above, this leads to an apparent shift of the period in the preasymptotic range, which is precisely what happens in this case. Let us attempt to estimate the value of $\Delta_{L}$. As seen from, e.g., Fig. 22 of Ref. $7, r_{\downarrow}$ increases with decreasing energy. Furthermore, as seen from Fig. 2 of Ref. 18 and Fig. 2 of Ref. 21, $r^{\downarrow}$ increases very strongly with $\mathbf{k}_{\|}$and full reflection is reached at a distance $0.1 \times \pi / a$ from $\bar{\Gamma}$; indeed, the low reflectivity arises only in a narrow window around $\bar{\Gamma}$. Taking these two contributions into account, we arrive at the result:

$$
\Delta_{L} \approx-\mathrm{i} \times 50 \mathrm{AL}
$$

Because $q_{L}^{\star}$ is negative, this result implies that the preasymptotic correction will lead to an apparent oscillation period in the preasymptotic regime that is shorter than the asymptotic one; this conclusion provides an consistent explanation for the discrepancy on the long oscillation period mentioned above. However, the preasymptotic regime here has an unusually large extension and asymptotic behavior is expected to hold only for $D \gg$ $50 \mathrm{AL}$; thus, the analysis of Sec. IVB, which was limited to the lowest order in $1 / D$, is certainly not sufficient to analyse the results obtained from experiments and from "exact" calculations and it is not surprising that not only the period itself, but also the amplitude of the long period oscillation predicted from the asymptotic approximation is inappropriate for spacer thicknesses $D \leq 50 \mathrm{AL}$.

Actually, $90 \%$ of the result (30) for $\Delta_{L}$ is due to the $\mathbf{k}_{\|}$ dependence of $r^{\downarrow}$, so that the unusually long preasymptotic regime for the long-period oscillation is to be attributed to the presence of a narrow window of low reflectivity near $\bar{\Gamma}$ in a region of otherwise total reflectivity, for minority spin. This pecularity can in turn be explained by a rather simple following argument: The relevant band in the $\mathrm{Cu}$ is mostly of $p_{z}$ character. The reflectivity is due to the hybridization of the corresponding $p_{z}$ band in Co with the minority spin $d$ bands. At the center $\bar{\Gamma}$ of the two-dimensional Brillouin zone, only the $d_{3 z^{2}-r^{2}}^{\downarrow}$ band is allow by symmetry to hybridize with the $p_{z}^{\downarrow}$ band, and hence to contribute to the reflectivity; however, because the $d_{3 z^{2}-r^{2}}^{\downarrow}$ band is almost full, it yields only a weak reflection coefficient at the Fermi level, and total reflection is attained only $0.5 \mathrm{eV}$ below $\varepsilon_{F}$. But, as one moves away for $\bar{\Gamma}$, the $d_{x z}^{\downarrow}$ and $d_{y z}^{\downarrow}$ bands that lie close to the Fermi level are allowed to hybridize with the $p_{z}^{\downarrow}$ band, which yields a strong increase of $r^{\downarrow}$ and, eventually, total reflection.

\section{CONCLUSION}

I have presented a detailed discussion of the question of preasymptotic corrections for the interlayer exchange coupling. A systematic method for computing exactly the preasymptotic corrections to arbitrary order in $D^{-1}$ has been presented, and the explicit expression of the first correction term (of order $D^{-3}$ ) has been given.

This method allows one to assess quantitatively the spacer thickness range in which the asymptotic approximation is expected to be reliable, and the one in which preasymptotic corrections should be taken into account. In the latter case, I have shown that the preasymptotic correction alters the amplitude and/or the apparent oscillation period, depending on the argument of the correction parameter $\Delta$.

The case of $\mathrm{Co} / \mathrm{Cu} / \mathrm{Co}(001)$ has been discussed in detail. I have shown that most of the discrepancy between the asymptotic approximation and "exact" calculations for the system can be understood on the basis of the theory for preasymptotic corrections presented in this paper. 


\section{APPENDIX:}

\section{Integration over energy}

We consider here the following integral

$$
I_{n}(T) \equiv \int_{-\infty}^{+\infty} \mathrm{d} \varepsilon f(\varepsilon, T) \varepsilon^{n} \exp (2 \mathrm{i} \varepsilon \alpha) .
$$

We can compute it by using the method of residues. The Fermi-Dirac function $f(\varepsilon, T)$ has poles for

$$
\varepsilon_{p}=\mathrm{i} \pi(2 p+1) \beta^{-1}
$$

where $p$ is an integer and $\beta \equiv\left(k_{B} T\right)^{-1}$. The corresponding residues are equal to $-\beta^{-1}$. Closing the integration path in the upper half of the complex plane, we obtain

$$
I_{n}(T)=-2\left(\frac{\mathrm{i} \pi}{\beta}\right)^{n+1} \sum_{p=0}^{+\infty}(2 p+1)^{n} \mathrm{e}^{-2 \pi(2 p+1) \alpha / \beta} .
$$

Finally, we obtain

$$
I_{n}(T)=I_{n}^{0} F_{n}\left(\frac{2 \pi \alpha}{\beta}\right)
$$

whith

$$
I_{n}^{0}=-\mathrm{i}^{n+1} \alpha^{-(n+1)} n !
$$

and

$$
F_{n}(x) \equiv \frac{(-1)^{n}}{n !} x^{n+1} \frac{\mathrm{d}^{n}}{\mathrm{~d} x^{n}}\left(\frac{1}{\sinh x}\right) .
$$

\section{Integration over wave vector}

Here we calculate the following integral.

$$
B_{2 n} \equiv \int_{-\infty}^{\infty} \mathrm{d} k k^{2 n} \exp \left(-\mathrm{i} \gamma k^{2}\right) .
$$

We can deform the integration path in the complex plane in such a way that the point $k \overline{-} 0$ be traversed along the direction of steepest descent 31 The integral is then easily calculated, and we get:

$$
B_{2 n}=\left(\frac{-\mathrm{i}}{\gamma}\right)^{n+1 / 2} \frac{(2 n+1) ! !}{2^{n}(2 n+1)} \sqrt{\pi} .
$$

${ }^{1}$ S.S.P. Parkin, N. More, and K.P. Roche, Phys. Rev. Lett. 64, 2304 (1990).

${ }^{2}$ K.B. Hathaway, A. Fert, P. Bruno, D.T. Pierce, J. Unguris, R.J. Celotta, and S.S.P. Parkin, in Ultrathin Magnetic Films, vol.2, edited by B. Heinrich and J.A.C. Bland (Springer-Verlag, Berlin, 1994), chap. 2, p. 45.

3 J.C. Slonczewski, J. Magn. Magn. Mater. 150, 13 (1994).

${ }^{4}$ D.M. Edwards, J. Mathon, R.B. Muniz, and M.S. Phan, Phys. Rev. Lett. 67, 493 (1991).

${ }^{5}$ P. Bruno, J. Magn. Magn. Mater. 121, 248 (1993).

${ }^{6}$ M.D. Stiles, Phys. Rev. B 48, 7238 (1993).

${ }^{7}$ P. Bruno, Phys. Rev. B 52, 411 (1995).

${ }^{8}$ P. Bruno and C. Chappert, Phys. Rev. Lett. 67, 1602 (1991); 67 2592(E) (1991).

${ }^{9}$ Y. Yafet, Phys. Rev. B 36, 3948 (1987).

${ }^{10}$ P. Bruno and C. Chappert, Phys. Rev. B 46261 (1992).

11 J. Unguris, R.J. Celotta, and D.T. Pierce, J. Appl. Phys. 75, 6437 (1994).

12 J. Unguris, R.J. Celotta, and D.T. Pierce, Phys. Rev. Lett. 79, 2734 (1998).

13 P. Lang, L. Nordström, R. Zeller, and P.H. Dederichs, Phys. Rev. Lett. 71, 1927 (1993).

${ }^{14}$ L. Nordström, P. Lang, R. Zeller, and P.H. Dederichs, Phys. Rev. B 50, 13058 (1994).

15 J. Kudrnovský, V. Drchal, I. Turek, and P. Weinberger, Phys. Rev. B 50, 16105 (1994).

${ }^{16}$ P. Lang, L. Nordström, K. Wildberger, R. Zeller, P.H. Dederichs, and T. Hoshino, Phys. Rev. B 53, 9092 (1996).

${ }^{17}$ V. Drchal, J. Kudrnovský, I. Turek, and P. Weinberger, Phys. Rev. B 53, 15036 (1996).

18 P. Bruno, J. Magn. Magn. Mater. 148, 202 (1995).

19 J. Mathon, M. Villeret, R.B. Muniz, J. d'Albuquerque e Castro, and D.M. Edwards, Phys. Rev. Lett. 74, 3696 (1995).

${ }^{20}$ B. Lee and Y.C. Chang, Phys. Rev. B 52, 3499 (1995).

${ }^{21}$ M.D. Stiles, J. Appl. Phys. 79, 5805 (1996).

22 J. d'Albuquerque e Castro, J. Mathon, M. Villeret, and A. Umerski, Phys. Rev. B 53, R13306 (1996).

23 J. Mathon, M. Villeret, A. Umerski, R.B. Muniz, J. d'Albuquerque e Castro, and D.M. Edwards, Phys. Rev. B 56, 11797 (1997).

24 J. Kudrnovsky (private communication).

${ }^{25}$ P. Bruno (pnpublished).

${ }^{26}$ As usual, 0 , $\mathrm{O}$ since $q^{\star}$ is defined only within a multiple of $2 \pi / d$ ( $d$ being the interplane distance in the normal direction), we take aliasing into account by making the choice that yields $-\pi / d \leq q^{\star} \leq+\pi / d$.

${ }^{27}$ M.T. Johnson, S.T. Purcell, N.W.E. McGee, R. Coehoorn, J. aan de Stegge, and W. Hoving, Phys. Rev. Lett. 68, 2688 (1992)

${ }^{28}$ P.H. Dederichs, K. Wildberger, and R. Zeller, Physica B 237-238, 239 (1997).

${ }^{29}$ P. Bruno, Europhys. Lett. 23, 615 (1993).

${ }^{30}$ P.J.H. Bloemen, M.T. Johnson, M.T.H. van de Vorst, R. Coehoorn, J.J. de Vries, R. Jungblut, J. aan de Stegge, A. Reinders, and W.J.M. de Jonge, Phys. Rev. Lett. 72, 764 (1994).

${ }^{31}$ J.W. Negele and H. Orland, Quantum Many-Particle Systems (Addison-Wesley, Redwood City, 1988), p. 121.

* Electronic address: bruno@mpi-halle.de 